\title{
Supramolecular Loop Stitches of Discrete Block Molecules on Graphite: Tunable Hydrophobicity by Naphthalenediimide End- Capped Oligodimethylsiloxane
}

\author{
José Augusto Berrocal, ${ }^{\dagger}$ Joan Teyssandier, ${ }^{\ddagger 0}$ Olga J. G. M. Goor, ${ }^{\dagger}$ Steven De Feyter, ${ }^{*} \neq$ \\ and E. W. Meijer*, ${ }^{*}(0)$ \\ ${ }^{\dagger}$ Institute for Complex Molecular Systems and Laboratory of Macromolecular and Organic Chemistry, Eindhoven University of \\ Technology, 5600 MB Eindhoven, The Netherlands \\ ${ }^{\ddagger}$ Division of Molecular Imaging and Photonics, Department of Chemistry, KU Leuven-University of Leuven, Celestijnenlaan 200 F, \\ B-3001 Leuven, Belgium
}

\section{Supporting Information}

\begin{abstract}
The noncovalent functionalization of surfaces has gained widespread interest in the scientific community, and it is progressively becoming an extremely productive research field offering brand new directions for both supramolecular and materials chemistry. As the end-groups often play a dominant role in the surface properties obtained, creating loops with endgroups only at the surface will lead to unexpected architectures and hence properties. Here we report the self-assembly of discrete block molecules-structures in-between block copolymers and liquid crystals - featuring oligodimethylsiloxanes (ODMS) end-

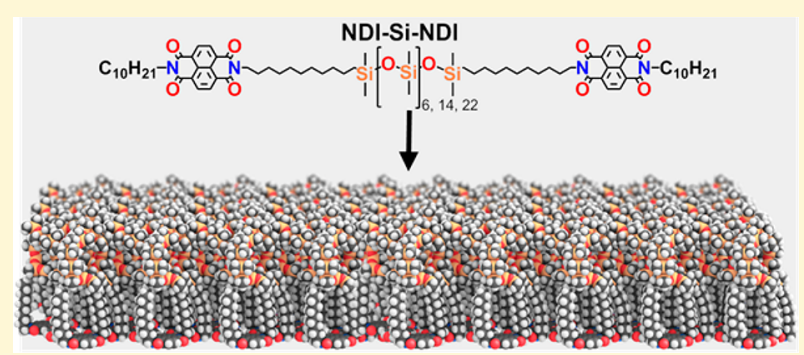
capped with naphthalenediimides (NDIs) at the 1-phenyloctane/highly oriented pyrolytic graphite (1-PO/HOPG) interface. These structures produce unprecedented vertically nanophase-separated monolayers featuring NDI moieties that regularly arrange on the HOPG surface, while the highly dynamic ODMS segments form loops above them. Such arrangement is preserved upon drying and generates hydrophobic HOPG substrates in which the ODMS block length tunes the hydrophobicity. Thus, the exact structural fidelity of the discrete macromolecules allows for the correlation of nanoscopic organization with macroscopic properties of the self-assembled materials. We present a general strategy for tunable hydrophobic coatings on graphite based on molecularly combining crystalline aromatic moieties and immiscible oligodimethylsiloxanes.
\end{abstract}

\section{INTRODUCTION}

The chemistry and physics of perfectly defined macromolecular architectures have recently generated rising interest as they bridge organic and polymer chemistries. ${ }^{1}$ This constructive cooperation between organic and polymer chemists resonates in a number of shared research topics, among which is materials science. $^{2}$ The connection between nanoscopic structure and macroscopic properties of a material is a well-established concept in the scientific community, but quantitative proof has not yet been fully demonstrated and the dispersity of polymeric structures significantly complicates this quantitative characterization. ${ }^{3}$ It is proposed that a more reliable correlation between nanostructure and macroscopic properties can be obtained from discrete macromolecules that self-assemble into precise, long-range ordered morphologies. ${ }^{4}$ In this case each structure is identical to its neighbors, and overall properties are not modulated by dispersity.

The currently blooming research topic of noncovalent surface functionalization is among the subjects that could benefit from discrete macromolecules. The high degree of organization of functional molecules in a physisorbed selfassembled monolayer is expected to furnish better control over surface properties, but the demonstration of this concept at the full surface scale (macroscale) is still awaited. ${ }^{5}$ This is particularly more challenging for the supramolecular functionalization of surfaces, where the competition between the many weak intermolecular interactions can result in a variety of kinetically trapped states.

The surface-supported assembly of perfectly defined small compounds ( $2 \mathrm{D}$ crystal engineering) has produced so far a multitude of structures and patterns where the molecular arrangement is controlled by the interplay of intermolecular and molecule-surface interactions. ${ }^{6,7}$ This strategy can produce very ordered arrays but possesses its limitations in terms of applicability. The latter can be improved by exploiting polymeric systems. Polymer-functionalized flat surfaces have been largely investigated by scanning probe microscopy techniques, especially in the case of conjugated polymers. ${ }^{8-12}$ However, the size dispersity and high molecular weight limit the long-range order of the $2 \mathrm{D}$ assemblies. ${ }^{13}$ It follows that

Received: February 24, 2018

Revised: April 30, 2018

Published: May 1, 2018 
Chart 1. Fully Extended Chemical Structures of Model NDIs 1 and 2 and NDI-Si8-NDI, NDI-Si16-NDI, and NDI-Si24-NDI Block Molecules

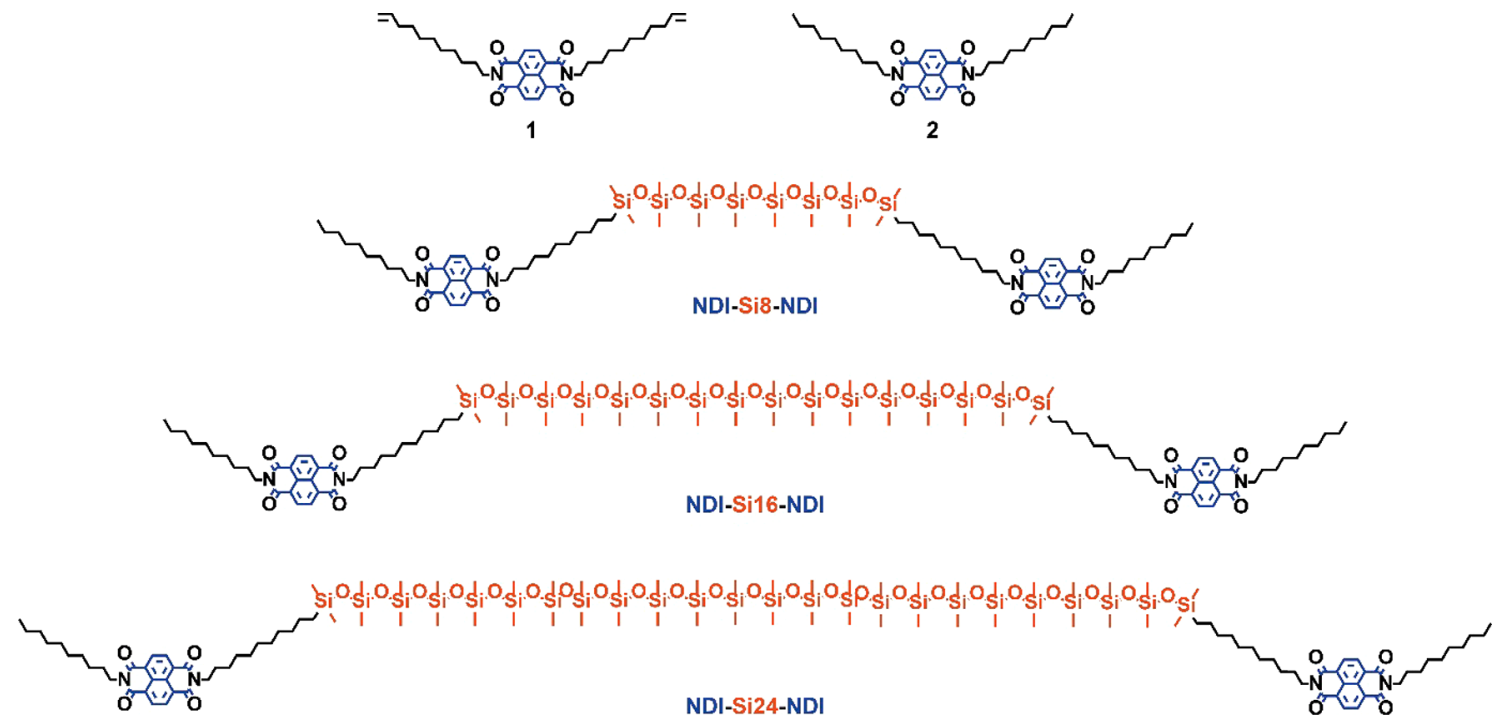

monodisperse oligomers ${ }^{14-16}$ may represent a reasonable solution to retain the advantages of both molecular systems. Another critical challenge for the elaboration of functional structures on surface is to control the projection of $2 \mathrm{D}$ assemblies in the third dimension. Indeed, growing upstanding functions (i.e., decoupled from the surface) on top of a physisorbed monolayer without interfering with its organization is being actively pursued. ${ }^{5,17-19}$ Consequently, attaching a functional oligomer with poor affinity for the surface to an anchoring moiety that will steer the assembly appears as a promising strategy for the design of functional adlayers.

Recent progress in the synthesis of discrete oligodimethylsiloxanes (ODMS) $)^{20,21}$ in our lab has allowed us to investigate the bulk self-assembly of defined macromolecules containing such thermodynamically immiscible units. By exploiting the large synthetic scope of hydrosylilation, ${ }^{22,23}$ we and others produced monodisperse block co-oligomeric architectures featuring oligo-lactic acid, ${ }^{20}$ ureidopyrimidinones (UPys), ${ }^{21}$ naphthalenediimides (NDIs), ${ }^{24}$ and azobenzenes (Azos). ${ }^{25,26}$ We found that when thermodynamic incompatibility is coupled to crystallinity, a synergy between the two driving forces leads to sub-10 nm periodically structured materials composed of crystalline domains (UPys, NDIs, Azos) dispersed into an amorphous ODMS matrix. ${ }^{24}$ We refer to such structures as block molecules and place them in-between liquid crystals ${ }^{27}$ and block copolymers. ${ }^{28-30}$

By extending the concepts of $2 \mathrm{D}$ supramolecular assembly to larger molecular dimensions, we aimed to produce highly controllable functional surfaces with tunable properties. Ogawa et al. have previously shown that bis- $n$-alkyl naphthalenediimide (NDI) derivatives form highly regular patterns at the 1tetradecene/HOPG interface. ${ }^{31}$ Either lamellar or hexagonal arrangements formed as the result of a delicate interplay between enthalpic and entropic forces associated with the alkyl chain length. ${ }^{31}$ Inspired by their work, we envisioned that connecting two "adhesive" end-groups (NDIs) via a "nonadhesive" linker (ODMS) may result in the formation of supramolecular loop stitches above the HOPG surface. We report the self-assembly of NDI-Si8-NDI, NDI-Si16-NDI, and NDI-Si24-NDI (Chart 1), which syntheses are reported elsewhere, $^{24}$ at the 1-phenyloctane/highly oriented pyrolytic graphite (1-PO/HOPG) interface and compare it to that of model compounds 1 and 2 . We show that the $2 \mathrm{D}$ self-assembly of our NDI-Si-NDI leads to unprecedented vertically nanophase-separated monolayers featuring highly regular NDI hexagonal patterns on graphite, while the ODMS chains are liquid-like. The latter form loops as a consequence of the particular NDI-Si-NDI design. Joining crystalline organic moieties (NDIs) to discrete oligomeric units (ODMS) brings to the perfect combination between high organization and flexibility. Such two contrasting features find a remarkable balance in our system, where we can achieve a high degree of organization of the NDIs underneath despite the presence of the flexible loops. Moreover, the exact molecular definition results in functional HOPG with the degree of hydrophobicity determined by the length of the ODMS block. Hence, we can efficiently correlate macroscopic properties and nanoscopic organizations of our materials by pursuing discrete (macro)molecular definition.

\section{RESULTS AND DISCUSSION}

Compounds Deposition on HOPG, STM Imaging, and Thin Film Structures. The structure of the self-assembled NDI-Si-NDI layers was investigated by in situ scanning tunneling microscopy (STM) imaging at the 1-PO/HOPG interface. After optimization of solution concentrations (see Supporting Information), STM revealed organized monolayers with complete HOPG coverage for all of the NDI-Si-NDI block molecules as well as for $\mathbf{1}$ (Figure 1). Model compound $\mathbf{1}$ self-assembled into a highly regular hexagonal porous network (Figure 1a). Its lattice parameters $(a=b=2.02 \pm 0.05 \mathrm{~nm}, \alpha=$ $60 \pm 1^{\circ}$ ) were in agreement with the so-called "honeycomb B" pattern observed by Ogawa et al. ${ }^{31}$ In this arrangement, the aromatic cores lay flat on HOPG and form a Kagome lattice, while the alkyl chains remain in solution (Figure 1b). Since the NDI-Si-NDI possess alkyl parts composed of 10 and 11 carbon atoms $\left(\mathrm{C}_{10}\right.$ and $\mathrm{C}_{11}$, respectively), model compound 2 was also imaged (Figure S1a). Patterns identical to those of $\mathbf{1}$ were obtained with this $\mathrm{C}_{10}$-NDI $(a=b=2.04 \pm 0.06 \mathrm{~nm}, \alpha=59 \pm$ $1^{\circ}$ ). 
(a)

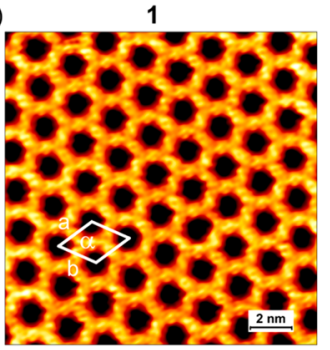

(c)

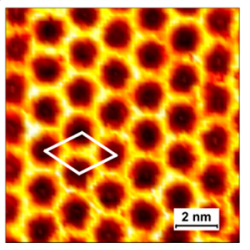

(d) NDI-Si16-ND

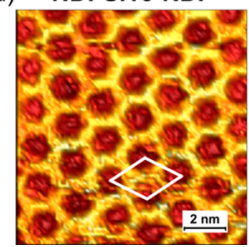

(e)

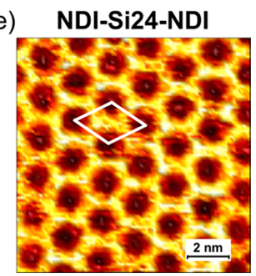

Figure 1. STM images of the hexagonal lattices of $1(a=b=2.02 \pm$ $\left.0.05 \mathrm{~nm}, a=60 \pm 1^{\circ}\right)$, NDI-Si8-NDI $(a=b=2.03 \pm 0.2 \mathrm{~nm}, a=61$ $\left.\pm 2^{\circ}\right)$, NDI-Si16-NDI $\left(a=b=2.04 \pm 0.2 \mathrm{~nm}, a=59 \pm 2^{\circ}\right)$, and NDI-Si24-NDI $\left(a=b=1.99 \pm 0.2 \mathrm{~nm}, a=61 \pm 3^{\circ}\right)$ at the 1-PO/ HOPG interface. (a) STM image of $1\left(C=10^{-3} \mathrm{M}\right)$ together with (b) a molecular model describing the arrangement of the NDI cores in the 2D network (the alkyl chains are not represented for sake of clarity). STM images of (c) NDI-Si8-NDI, (d) NDI-Si16-NDI, and (e) NDISi24-NDI ( $C=10^{-4} \mathrm{M}$ for all three compounds). Imaging parameters: (a) $I_{\text {set }}=350 \mathrm{pA}, V_{\text {bias }}=1.65 \mathrm{~V}$; (c) $I_{\text {set }}=50 \mathrm{pA}, V_{\text {bias }}=1.6 \mathrm{~V}$; (d) $I_{\text {set }}=$ $50 \mathrm{pA}, V_{\text {bias }}=2.0 \mathrm{~V}$; (e) $I_{\text {set }}=60 \mathrm{pA}, V_{\text {bias }}=2.0 \mathrm{~V}$.

Intriguingly, the three NDI-Si-NDI exhibited the same hexagonal pattern as $\mathbf{1}$ and 2, regardless of the presence and length of the siloxane chains (Figure $1 \mathrm{c}-\mathrm{e}$ ). This suggests that the ODMS block stays in solution similarly to the alkyl chains in $\mathbf{1}$ and 2. Moreover, the identical organization shown by all five molecules (Chart 1) points to a lack of ODMS influence on the self-assembly process. Hence, the monolayer formation must be controlled by the remaining components of the blocky architectures, namely the NDI moieties and alkyl chains.

Previous literature ${ }^{31,32}$ revealed that the HOPG-NDI $\pi-\pi$ interaction is highly enthalpically favored and results in complete adhesion of the aromatic cores to the conjugated surface. The HOPG-alkyl chain interaction, instead, only relies on weak, yet favorable, forces that compete with the entropic penalty for freezing the rotation around $\mathrm{C}-\mathrm{C}$ bonds and stretching the carbon chain. ${ }^{31}$ Such competition generated three regimes associated with the carbon chain length. Short $\left(\mathrm{C}_{n}\right.$, with $\left.n \leq 4\right)$ or long $\left(\mathrm{C}_{n}\right.$, with $\left.n \geq 13\right)$ carbon chains fully adhered to the HOPG and gave place to lamellar arrangements because enthalpically favored. ${ }^{31}$ By contrast, the entropic penalty in the intermediate alkyl chain regime $\left(\mathrm{C}_{n}\right.$, with $4 \leq$ $n \leq 12$ ) forced only the aromatic cores to lay on the surface, while the alkyl chains remained upright and moved "freely" in solution. Such molecular arrangement generated several hexagonal porous morphologies (honeycomb A, B, and C) at the tetradecene/HOPG interface. ${ }^{31,32}$ The nonadherence of the alkyl chains in this intermediate regime also resulted in similar lattice parameters independent of the chain length, ${ }^{31}$ which is strikingly similar to the (absence of) influence of the ODMS in our system.

The only part of the NDI-Si-NDI observed by STM is the NDI block, which corresponds to the bright contrast areas in Figure 1. Since the rest of the molecule is completely invisible, we hypothesized monolayers composed by NDI cores arranged flat on HOPG, while the alkyl spacers and ODMS avoid contact with the surface and remain in solution (Figure 2). Whereas the
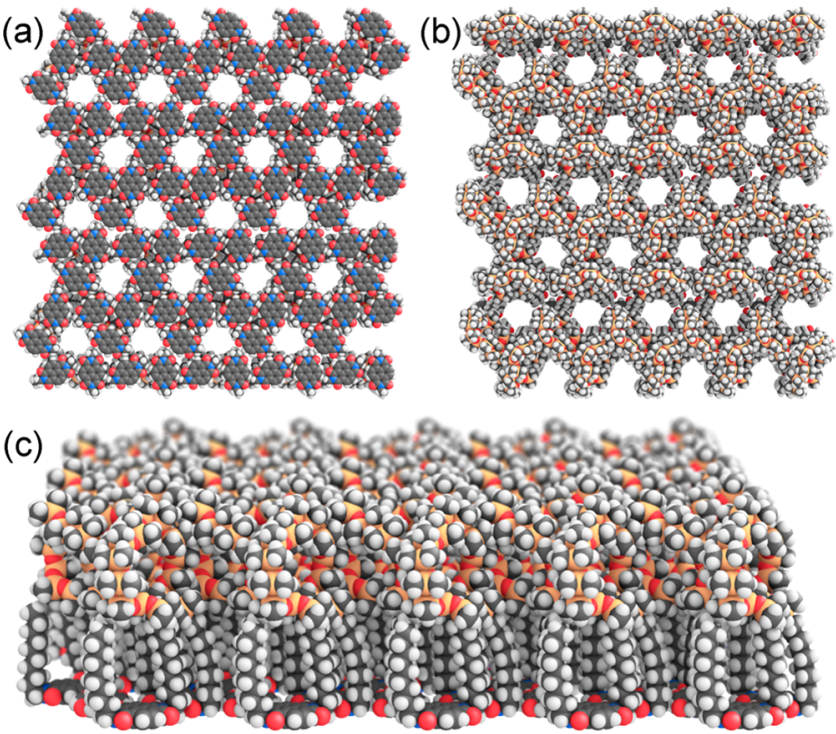

Figure 2. View from the bottom (a), top (b), and side (c) of the hypothesized structure of the hexagonal lattices of the NDI-Si-NDI. Carbon atoms are depicted in gray, nitrogen atoms in blue, oxygen atoms in red, hydrogen atoms in white, and silicon atoms in orange.

upright configuration has already been reported for alkyl chains in differently designed NDI systems, ${ }^{31,32}$ it is also reasonable for highly thermodynamically immiscible units such as the ODMS. The high incompatibility of the ODMS represents an additional enthalpic driving force, next to the previously discussed entropic penalty, to keep the $\mathrm{C}_{11}$-spacers distant from the graphite surface.

Polydimethylsiloxanes (PDMS) are frequently applied as surface wetting agents because of their low surface energy. ${ }^{33,34}$ They are typically end-capped with polar functional groups $\left(-\mathrm{OH},-\mathrm{NH}_{2}\right)$ to favor thin film deposition on previously cleaned (e.g., piranha treatment, $\mathrm{O}_{2}$ plasma etching, UV ozone treatment) glass or silica. In stark contrast, we have observed a very low wetting ability by our monodisperse ODMS (vide infra), most likely due to a stronger thermodynamic incompatibility at this particular molecular weight regime. Only one report on the full deposition of ODMS-based block molecules on patterned silicon wafers has been published. ${ }^{25}$ PDMS surface coatings were pivotal to enhance the wettability of the wafers by the ODMS before thin film deposition. ${ }^{25}$ Congruously, no literature is available about ODMS interacting with HOPG substrates.

We hypothesized the presence of ODMS loops above the NDIs because of the particular NDI-Si-NDI design and the highly favorable NDI-HOPG adhesive forces ${ }^{31,32}$ (Figure 2). The peculiar ODMS configuration is favored by their extraordinary flexibility deriving from longer $\mathrm{Si}-\mathrm{O}$ bond length and larger $\mathrm{Si}-\mathrm{O}-\mathrm{Si}$ dihedral angles ${ }^{35}$ compared to oligoethylene and oligoethylene oxide chains. Thus, the ODMS form loops because of very low strain (enthalpy) and entropic penalty. Despite the well-ordered NDI hexagonal lattice on the bottom part of the monolayer, it is very unlikely that such order is preserved also on the top "loopy" ODMS layer. A number of different conformations of the ODMS backbone, as well as an elevated number of spatial combinations between the NDI 


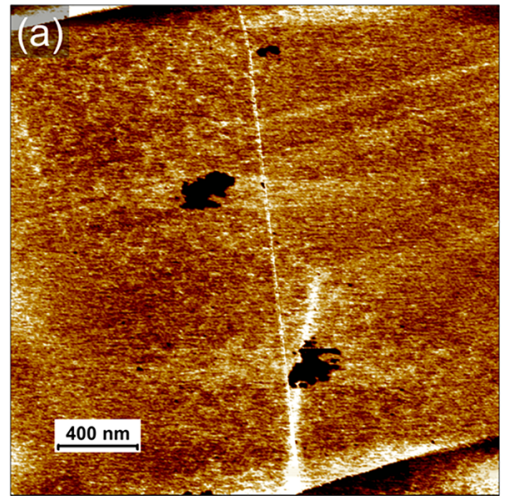

(b)

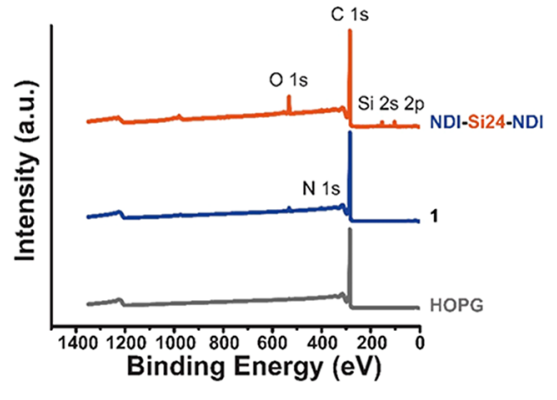

Figure 3. (a) AFM topography image of a dry film of 1 on HOPG. (b) XPS profiles of "control HOPG" (gray trace) and HOPG substrates modified with 1 (blue trace) and NDI-Si24-NDI (orange trace).

submonolayer and ODMS top-layer, are possible by the same argument of the ODMS flexibility. However, it remains remarkable that the surface shows a regular thermodynamically stable NDI pattern, and no kinetic traps due to unfavorable loops are observed. Although the images reported in Figure 2 are a simplified pictorial representation of the hypothesized structure, it should be stressed that this hypothesis is in qualitative agreement with our previous investigation on the bulk self-assembly of the NDI-ODMS conjugates. ${ }^{24}$ While the rigid aromatic block forms extremely well-ordered domains, the soft and flexible ODMS component prefers an amorphous organization. In-between these two units, the alkyl spacers act as connecting linkers and guide through the transition from order to disorder or vice versa. ${ }^{24}$

2.2. Structure Verification with AFM and XPS. To verify the hypothesized NDI-Si-NDI spatial arrangement at the 1PO/HOPG interface, as well as to exclude the possibility of a siloxane-free impurity as the cause of the STM observations (see also Supporting Information, pp 3 and 4), we used tapping-mode atomic force microscopy (AFM) and X-ray photoelectron spectroscopy (XPS). Both techniques can be applied on dry and wet samples - with certain limitations in the presence of solvents-but we opted for the characterization of the dry states to support the water contact angle (WCA) measurements presented in the next section (vide infra). Since the arrangement shown in Figure 2 is sustained by the solvent, a collapse of the ODMS loops on top of the NDIs is expected. Nevertheless, the layered structure should be preserved in the absence of dewetting. An example of monolayer dry film of $\mathbf{1}$ is illustrated by the topography image shown in Figure 3a. Images of the other compounds are shown in Figure S6. All films display homogeneous morphology, exhibit very few defects (holes or cracks in the layer), and possess roughness similar to the underlying graphite. STM at the air/HOPG interface revealed retention of the hexagonal organization even in the dry state (Figure S4). Scratching (nanoshaving) of the obtained films was performed to compare thicknesses and ensure single layer formation. The line profile taken from the subsequent image allows to measure precisely the difference in height between the film and HOPG (Figure S9). The value for compound 1 is $5.0 \pm 0.7 \AA$, which is highly compatible with a monolayer of this molecule. For the NDI-Si-NDI, the thicknesses were $5.8 \pm 0.6 \AA$ (Si8), $4.9 \pm 0.5 \AA$ (Si16), and $5.0 \pm 1.3 \AA$ (Si24). Therefore, the thickness of the films of each compound is surprisingly the same and clearly points to monolayer formation in all cases.
As previously discussed for the STM measurements, a collapse of the alkyl chains and ODMS on the bottom NDI layer is expected in the dry samples. In the case of compound $\mathbf{1}$, the collapsed alkyl chains cover the empty pores and/or the underlying NDI cores. We speculate that the $\mathrm{C}_{11}$ spacers form a rather disordered top layer, with a "corrugated" profile characterized by irregular voids. The thickness measured by AFM should correspond to the NDI + alkyl chain areas (the alleged variations in height occur over a too small distance to be detected by the tip). For the NDI-Si-NDI, the additional ODMS block has to be taken into account. In line with our previous investigation on the NDI-ODMS conjugates, ${ }^{24}$ we expect the ODMS to act as "space fillers" also in this case. The voids created by the alkyl chains of $\mathbf{1}$ are filled by amorphous and flexible siloxanes, which ultimately create a hydrophobic top layer. Hence, the spreadable liquid nature of the ODMS is observed also in the case of the NDI-Si-NDI on HOPG. While liquids typically distribute on substrates as a result of favorable adhesive forces, in the current system the ODMS cover the underlying layer only due to a (chemical) design constraint. The consistent film thicknesses of the NDI-Si-NDI series can be inferred from this molecular picture: the small addition of 8 siloxane units-moreover in a spread configuration-is too sensitive to be detected with the accuracy of the AFM technique. This limitation raises a pivotal technological challenge to advance the field: defined macromolecular structures that self-assemble into precise nanomorphologies are finally available, but the arsenal of experimental techniques featuring (sub)molecular resolution is too limited.

The qualitative indications obtained from the AFM measurements were further confirmed with X-ray photoelectron spectroscopy (XPS). A “control HOPG” sample, freshly cleaved HOPG treated with 1-PO, was also measured as reference. The XPS measurements revealed distinct differences between the various surfaces (Figure $3 \mathrm{~b}$; Table $\mathrm{S} 1$ and Figure S10). As expected, the control HOPG sample was mainly composed of carbon (Figure 3b, gray trace). A gradual enrichment in both nitrogen and oxygen content was observed upon HOPG modification with $\mathbf{1}$, which is consistent with the chemical structure of the NDI derivative (Figure 3b, blue trace). Finally, the XPS spectrum of NDI-Si24-NDI revealed the presence of $\mathrm{Si}$ as additional surface component (Figure $3 \mathrm{~b}$, orange trace). Consistent results were obtained with NDI-Si8-NDI and NDISi16-NDI (Table S1 and Figure S10), which excluded the possible presence of identical contaminants in all modified substrates. Furthermore, the content in $\mathrm{Si}$ increases pro- 
gressively with the increasing length of the ODMS chain, demonstrating a tunable composition of the adlayer.

We further studied the HOPG surfaces with angle-resolved XPS (ARXPS) by scanning the modified substrates from $0^{\circ}$ to $75^{\circ}$ with angle increments of $15^{\circ}$ (Table 1 ). The overall signal

Table 1. Angle-Resolved XPS Measurements on HOPG Modified with NDI-Si24-NDI with Surface Percentage Composition ([\%]) for Carbon (C), Nitrogen (N), Oxygen $(\mathrm{O})$, and Silicon $(\mathrm{Si})$

\begin{tabular}{clccc} 
angle $[\mathrm{deg}]$ & $\mathrm{C}[\%]$ & $\mathrm{N}[\%]$ & $\mathrm{O}[\%]$ & $\mathrm{Si}[\%]$ \\
\hline 0 & 87.38 & 0.57 & 6.58 & 5.45 \\
15 & 86.9 & 0.71 & 6.72 & 5.67 \\
30 & 85.68 & 0.55 & 7.55 & 6.22 \\
45 & 82.74 & 0.55 & 8.95 & 7.75 \\
60 & 78.3 & 0.73 & 11.11 & 9.86 \\
75 & 73.09 & 2.23 & 12.71 & 11.97 \\
\hline
\end{tabular}

intensity of the NDI-Si24-NDI surface decreased upon increasing the tilting angle because of the lower penetration of the incident X-rays on the monolayer. The relative surface composition proved to be particularly sensitive to the increasing resolving angle, with a gradual increase in Si content upon increasing resolving angle. This corroborates the hypothesized enrichment in ODMS at the material surface, as the increasing relative intensity of the Si peaks strongly suggests the dominant role of the ODMS at the extreme surface. Similarly to the previous XPS measurements at $0^{\circ}$, also ARXPS provided consistent results within the NDI-Si-NDI series. The same qualitative trend in increasing $\mathrm{Si}$ content was witnessed with NDI-Si8-NDI and NDI-Si16-NDI upon tilting the sample (Tables S2 and S3).

2.3. Probing Macroscopic Properties of Nanoscopic Features. The hypothesized vertical nanophase separation of the NDI-Si-NDI monolayers should macroscopically result in hydrophobicity. Furthermore, the latter should be tunable via the ODMS length. This aspect is particularly interesting for applications, since contriving new strategies and concepts for extended and mechanically stable hydrophobic coatings is a current target of surface chemistry. ${ }^{36,37}$ We assessed the hydrophobicity of the NDI-Si-NDI-functionalized HOPG with water contact angle (WCA) and compared it to reference NDI 1 and control HOPG (Figure 4). HOPG modification

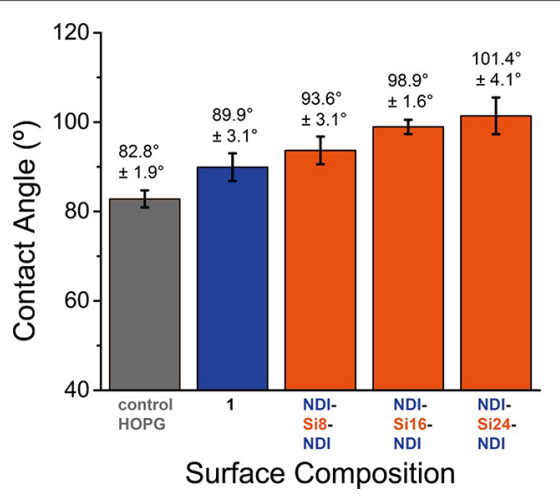

Figure 4. Water contact angle measurements performed on control HOPG and dry HOPG substrates modified with 1, NDI-Si8-NDI, NDI-Si16-NDI, and NDI-Si24-NDI. A trend in increasing hydrophobicity is observed upon extending the ODMS length of the NDISi-NDI. with 1 and the simple treatment with $1-\mathrm{PO}$ conferred an increase in hydrophobicity: compared to the $70^{\circ}$ WCA of pristine HOPG, values as high as $90^{\circ}$ and $83^{\circ}$ were measured for 1 and control HOPG, respectively (Figure 4 and Table S5). Remarkable increases in WCAs were obtained with the NDI-SiNDI, where modification with NDI-Si8-NDI, NDI-Si16-NDI, and NDI-Si24-NDI resulted in WCA equal to $94^{\circ}, 99^{\circ}$, and $101^{\circ}$, respectively (Figure 4 and Table S5). Such measurements corroborate the vertical nanophase separation in both wet and dry state, with the hydrophobic siloxane layer facing outward. They also highlight that the "simple" addition of our discrete ODMS to the "basic" NDI-alkyl motif is responsible for an $11^{\circ}$ WCA increase. A qualitative trend in increasing hydrophobicity was also observed with the NDI-Si-NDI series upon extending the ODMS length. While the first extension of 8 siloxane units (from $\mathrm{Si8}$ to $\mathrm{Si16}$ ) caused a more pronounced variation of the WCA measured, the second increment seemed to be more contained. We propose that further extension of the ODMS could result in asymptotic growth to the value typically reported for polydisperse PDMS-modified surfaces, approximately $110^{\circ} .^{38,39}$ The exact definition of our block molecules ensures consistent hydrophobicity throughout the entire substrate unlike polymeric samples, provided surface coverage is quantitative.

Finally, we stress the generality of our approach for the ODMS functionalization of HOPG. Aromatic moieties that show favorable adhesive interactions with graphite are necessary in the molecular design. The rational design allows to control the pattern formed on the top graphite layer as well as the position of the ODMS on the surface. We speculate that the richness of supramolecular moieties for surface chemistry $^{40-42}$ will allow the elaboration of other networks with different density and controllable patterns.

\section{CONCLUSIONS}

We reported the self-assembly of NDI-Si-NDI block molecules at the interface between graphite and 1-phenyloctane. Our discrete macromolecules give rise to a unique combination of high order and flexibility, surprisingly avoiding kinetic traps in the self-assembly process. STM analysis revealed the consistent generation of hexagonal patterns deriving from a delicate balance between favorable and unfavorable adhesive forces. We hypothesized unprecedented vertically nanophase-separated monolayers composed of flat NDI moieties neatly arranged on the top HOPG layer and irregular ODMS loops above it. This hypothesis is in qualitative agreement with our previous investigation on the bulk self-assembly of the NDI-ODMS conjugates: the aromatic blocks form crystalline domains, while the siloxanes are amorphous and fill space. The proposed structure was confirmed with AFM, XPS, and WCA. The architectures obtained are remarkable from both self-assembly and materials properties perspectives. On one hand, it is notable that the highly regular NDI arrays are not disturbed/ affected by the atop loops formed by our molecules in their surface architecture. On the other hand, the anchoring role of the end-groups ensures homogeneous materials properties throughout the whole surface, which are controlled by the ODMS loops. The exact molecular definition proved to be pivotal in tuning the hydrophobicity of the modified substrates. A convincing qualitative agreement between ODMS block length and hydrophobicity was measured for the dry HOPG surfaces modified with the NDI-Si-NDI, which allowed to correlate nanoscopic organization and macroscopic properties 
of our materials. We presented a suitable strategy for the preparation of hydrophobic coatings on graphite, a strategy molecularly translated into the "discrete" combination between crystalline aromatic moieties and highly immiscible oligodimethylsiloxanes. By exporting the emerging field of block molecules to graphite, we merged the best characters of $2 \mathrm{D}$ crystal engineering and polymer functionalization of substrates en route to generate highly controlled functional surfaces.

\section{EXPERIMENTAL SECTION}

Materials. Unless stated otherwise, all reagents and chemicals were obtained from commercial sources at the highest purity available and used without further purification. 1-Phenyloctane (Sigma, 98\%) and ethanol (Merck, >99.9\%) were used as received. All solutions were prepared in 1-phenyloctane (1-PO).

Scanning Tunneling Microscopy. In situ scanning tunneling microscopy (STM) experiments were carried out at room temperature $\left(21-23{ }^{\circ} \mathrm{C}\right)$ at the $1-\mathrm{PO} / \mathrm{HOPG}$ interface using a Multimode Nanoscope III-d STM (Veeco) and a PicoSPM (Agilent) instruments operating in constant-current mode. Prior to imaging, a drop of solution was placed onto a freshly cleaved surface of highly oriented pyrolytic graphite (HOPG, grade ZYB, Advanced Ceramics Inc., Cleveland, OH). STM tips were prepared by mechanical cutting from $\mathrm{Pt} / \mathrm{Ir}$ wire $(80 \% / 20 \%$, diameter $0.2 \mathrm{~mm})$. The imaging parameters are indicated in figure captions: sample bias $\left(V_{\text {bias }}\right)$ and tunneling current $\left(I_{\text {set }}\right)$. An organized monolayer could be observed in a range of concentrations between $5 \times 10^{-4}$ and $10^{-3} \mathrm{M}$ for 1 and between $10^{-5}$ and $5 \times 10^{-4} \mathrm{M}$ for the ODMS-containing compounds. For analysis purposes, recording of a monolayer image was followed by imaging the graphite substrate underneath it under the same experimental conditions, except for increasing the current and the lowering the bias. The images were corrected for drift via Scanning Probe Image Processor (SPIP) software (Image Metrology ApS), using the recorded graphite images for calibration purposes, allowing a more accurate unit cell determination. The images presented in Figure 1 are Gaussian filtered.

Atomic Force Microscopy. Dry films were prepared using the socalled "drop-coating" method. It consists of the deposition on HOPG of a droplet of a solution in 1-PO that is blown away by a $\mathrm{N}_{2}$ flow a few seconds later. Samples are then rinsed thoroughly in ethanol and blow dried again in $\mathrm{N}_{2}$. A $15 \mathrm{~min}$ postannealing at $90{ }^{\circ} \mathrm{C}$ was performed to ensure an optimal organization of the films. The coverage and layer thickness can be controlled by the concentration of the solution deposited on the surface. Concentrations similar to those giving rise to the organized networks observed by STM were used to obtain monolayers. These "ideal" concentrations are respectively $10^{-3}$ $\mathrm{M}$ for $1,3.5 \times 10^{-4} \mathrm{M}$ for NDI-Si8-NDI, $10^{-4} \mathrm{M}$ for NDI-Si16-NDI, and $7 \times 10^{-5} \mathrm{M}$ for NDI-Si24-NDI. To confirm the monolayer films are organized in the same pattern as in 1-PO, the dry samples were imaged using STM, in the same conditions as described above, but at the air/HOPG interface. AFM measurements were carried out using a PicoSPM (Agilent) machine under ambient conditions with silicon cantilevers (AC240TS, Olympus). Layer thickness was measured after mechanical scratching of the film in a defined square using AFM. In this method, molecules are removed by sweeping them away with the AFM tip, while the machine is operated in contact mode.

STM and AFM images were processed using the WSxM (Nanotec Electronica, Spain) software. $^{43}$ The molecular model provided in Figure $1 \mathrm{~b}$ was built using Hyperchem Professional 7.5.

X-ray Photoelectron Spectroscopy. X-ray photoelectron spectroscopy was performed on spin-coated coverslips using a Thermo Scientific K-alpha spectrometer equipped with a monochromatic, small-spot X261 ray source and a $180^{\circ}$ double focusing hemispherical analyzer with a 128-channel detector. The coverslips were mounted on the holder using carbon tape, and an aluminum anode ( $\mathrm{Al} \mathrm{K} \alpha, 1486.6$ $\mathrm{eV}, 72 \mathrm{~W}$ ) was used to obtain the spectra. Analysis and quantification of the spectra were performed using CasaXPS software version 2.3.16, using the $\mathrm{C} 1 \mathrm{~s}, \mathrm{~N} 1 \mathrm{~s}, \mathrm{O} 1 \mathrm{~s}$, and $\mathrm{Si} 2 \mathrm{p}$ regions.
Water Contact Angle. Water contact angles were measured on spin-coated surfaces at room temperature in air using an OCA 30 (DataPhysics). Deionized water droplets of $4 \mu \mathrm{L}$ were applied on the surface of the functionalized substrates. Images were recorded at a rate of 2.5 frames/s, and the angle at the surface-air-water interface was measured after $5 \mathrm{~s}$ using an automatic fitting routine (SCA20 software).

\section{ASSOCIATED CONTENT}

\section{Supporting Information}

The Supporting Information is available free of charge on the ACS Publications website at DOI: 10.1021/acs.chemmater.8b00820.

In situ STM imaging at the liquid/solid interface, X-ray photoelectron spectroscopy (XPS) measurements, angleresolved X-ray photoelectron spectroscopy (ARXPS) measurements, and water contact angle (WCA) experiments (PDF)

\section{AUTHOR INFORMATION}

\section{Corresponding Authors}

*E-mail e.w.meijer@tue.nl (E.W.M.).

*E-mail steven.defeyter@kuleuven.be (S.D.F.).

ORCID *

Joan Teyssandier: 0000-0003-4369-0542

E. W. Meijer: 0000-0003-4126-7492

\section{Author Contributions}

J.A.B. and J.T contributed equally.

\section{Notes}

The authors declare no competing financial interest.

\section{ACKNOWLEDGMENTS}

J.A.B., O.J.G.M.G., and E.W.M. thank the Dutch Ministry of Education, Culture and Science (Gravity program 024.001.035) and The Netherlands Organization for Scientific Research (NWO TOP-PUNT 718.014.003) for financial support. J.T. and S.D.F. acknowledge KU Leuven-Internal Funds, Fund of Scientific Research-Flanders (FWO), and the Belgian Federal Science Policy Office (IAP-7/05). The ICMS (Institute for Complex Molecular System) Animation Studio is acknowledged for providing the cartoons of the functionalized HOPG substrates. Mr. M. W. G. M. (Tiny) Verhoeven is acknowledged for helping with the XPS measurements.

\section{REFERENCES}

(1) Lutz, J.-F.; Lehn, J.-M.; Meijer, E. W.; Matyjaszewski, K. From Precision Polymers to Complex Materials and Systems. Nat. Rev. Mater. 2016, 1, 16024.

(2) Hawker, C. J.; Wooley, K. L. The Convergence of Synthetic Organic and Polymer Chemistries. Science 2005, 309, 1200-1205.

(3) van Krevelen, D. W.; te Nijenhuis, K. Properties of Polymers: Their Correlation with Chemical Structure; Their Numerical Estimation and Prediction from Additive Group Contributions; Elsevier: 2009.

(4) Aida, T.; Meijer, E. W.; Stupp, S. I. Functional Supramolecular Polymers. Science 2012, 335, 813-817.

(5) Sosa-Vargas, L.; Kim, E.; Attias, A.-J. Beyond "Decorative" 2D Supramolecular Self-Assembly: Strategies towards Functional Surfaces for Nanotechnology. Mater. Horiz. 2017, 4, 570-583.

(6) Elemans, J. A. A. W.; Lei, S.; De Feyter, S. Molecular and Supramolecular Networks on Surfaces: From Two-Dimensional Crystal Engineering to Reactivity. Angew. Chem., Int. Ed. 2009, 48, $7298-7332$ 
(7) Ciesielski, A.; Palma, C.-A.; Bonini, M.; Samorì, P. Towards Supramolecular Engineering of Functional Nanomaterials: PreProgramming Multi-Component 2D Self-Assembly at Solid-Liquid Interfaces. Adv. Mater. 2010, 22, 3506-3520.

(8) Samorí, P.; Francke, V.; Müllen, K.; Rabe, J. P. Self-Assembly of a Conjugated Polymer: From Molecular Rods to a Nanoribbon Architecture with Molecular Dimensions. Chem. - Eur. J. 1999, 5, 2312-2317.

(9) Mena-Osteritz, E.; Meyer, A.; Langeveld-Voss, B. M. W.; Janssen, R. A. J.; Meijer, E. W.; Bäuerle, P. Two-Dimensional Crystals of Poly(3-Alkyl-Thiophene)s: Direct Visualization of Polymers Folds in Submolecular Resolution. Angew. Chem., Int. Ed. 2000, 39, 2679-2684.

(10) Grévin, B.; Rannou, P.; Payerne, R.; Pron, A.; Travers, J. P. Multi-scale Scanning Tunneling Microscopy Imaging of Self-Organized Regioregular Poly(3-Hexylthiophene) Films. J. Chem. Phys. 2003, 118, 7097-7102.

(11) Bocheux, A.; Tahar-Djebbar, I.; Fiorini-Debuisschert, C.; Douillard, L.; Mathevet, F.; Attias, A.-J.; Charra, F. Self-Templating Polythiophene Derivatives: Electronic Decoupling of Conjugated Strands through Staggered Packing. Langmuir 2011, 27, 1025110255 .

(12) Saywell, A.; Sprafke, J. K.; Esdaile, L. J.; Britton, A. J.; Rienzo, A.; Anderson, H. L.; O'Shea, J. N.; Beton, P. H. Conformation and Packing of Porphyrin Polymer Chains Deposited Using Electrospray on a Gold Surface. Angew. Chem., Int. Ed. 2010, 49, 9136-9139.

(13) Xu, L.; Yang, L.; Lei, S. Self-Assembly of Conjugated Oligomers and Polymers at the Interface: Structure and Properties. Nanoscale 2012, 4, 4399-4415.

(14) Azumi, R.; Götz, G.; Debaerdemaeker, T.; Bäuerle, P. Coincidence of the Molecular Organization of $\beta$-Substituted Oligothiophenes in Two-Dimensional Layers and Three-Dimensional Crystals. Chem. - Eur. J. 2000, 6, 735-744.

(15) Mena-Osteritz, E.; Bäuerle, P. Complexation of $\mathrm{C}_{60}$ on a Cyclothiophene Monolayer Template. Adv. Mater. 2006, 18, 447-451.

(16) Gong, J.-R.; Yan, H.-J.; Yuan, Q.-H.; Xu, L.-P.; Bo, Z.-S.; Wan, L.-J. Controllable Distribution of Single Molecules and Peptides within Oligomer Template Investigated by STM. J. Am. Chem. Soc. 2006, 128, 12384-12385.

(17) Le Liepvre, S.; Du, P.; Kreher, D.; Mathevet, F.; Attias, A.-J.; Fiorini-Debuisschert, C.; Douillard, L.; Charra, F. Fluorescent SelfAssembled Molecular Monolayer on Graphene. ACS Photonics 2016, 3, 2291-2296.

(18) Visser, J.; Katsonis, N.; Vicario, J.; Feringa, B. L. TwoDimensional Molecular Patterning by Surface-Enhanced Zn-Porphyrin Coordination. Langmuir 2009, 25, 5980-5985.

(19) Baisch, B.; Raffa, D.; Jung, U.; Magnussen, O. M.; Nicolas, C.; Lacour, J.; Kubitschke, J.; Herges, R. Mounting Freestanding Molecular Functions onto Surfaces: The Platform Approach. J. Am. Chem. Soc. 2009, 131, 442-443.

(20) van Genabeek, B.; De Waal, B. F. M.; Gosens, M. M. J.; Pitet, L. M.; Palmans, A. R. A.; Meijer, E. W. Synthesis and Self-Assembly of Discrete Dimethylsiloxane-Lactic Acid Diblock Co-oligomers: The Dononacontamer and Its Shorter Homologues. J. Am. Chem. Soc. 2016, 138, 4210-4218.

(21) Zha, R. H.; de Waal, B.; Lutz, M.; Teunissen, A. J. P.; Meijer, E. W. End Groups of Functionalized Siloxane Oligomers Direct BlockCopolymeric or Liquid-Crystalline Self-Assembly Behavior. J. Am. Chem. Soc. 2016, 138, 5693-5698.

(22) Markó, I. E.; Stérin, S.; Buisine, O.; Mignani, G.; Branlard, P.; Tinant, B.; Declercq, J.-P. Selective and Efficient Platinum(0)-Carbene Complexes as Hydrosilylation Catalysts. Science 2002, 298, 204-206.

(23) De Bo, G.; Berthon-Gelloz, G.; Tinant, B.; Markó, I. E. Hydrosilylation of Alkynes Mediated by N-Heterocyclic Carbene Platinum (0) Complexes. Organometallics 2006, 25, 1881-1890.

(24) Berrocal, J. A.; Zha, R. H.; De Waal, B. F. M.; Lugger, J. A. M.; Lutz, M.; Meijer, E. W. Unraveling the Driving Forces in the SelfAssembly of Monodisperse Naphthalenediimide-Oligodimethylsiloxane Block Molecules. ACS Nano 2017, 11, 3733-3741.
(25) Nickmans, K.; Murphy, J. N.; de Waal, B.; Leclère, P.; Doise, J.; Gronheid, R.; Broer, D. J.; Schenning, A. P. H. J. Sub-5 nm Patterning by Directed Self-Assembly of Oligo (Dimethylsiloxane) Liquid Crystal Thin Films. Adv. Mater. 2016, 28, 10068-10072.

(26) Zha, R. H.; Vantomme, G.; Berrocal, J. A.; Gosens, R.; de Waal, B.; Meskers, S.; Meijer, E. W. Photoswitchable Nanomaterials Based on Hierarchically Organized Siloxane Oligomers. Adv. Funct. Mater. 2018, 28, 1703952 .

(27) Goodby, J. W. G.; Collings, P. J.; Kato, T.; Tschierske, C.; Gleeson, H. F.; Raynes, E. P. Handbook of Liquid Crystals; Wiley-VCH: 2014.

(28) Khandpur, A. K.; Foerster, S.; Bates, F. S.; Hamley, I. W.; Ryan, A. J.; Bras, W.; Almdal, K.; Mortensen, K. Polyisoprene-Polystyrene Diblock Copolymer Phase Diagram near the Order-Disorder Transition. Macromolecules 1995, 28, 8796-8806.

(29) Matsen, M. W.; Bates, F. S. Unifying Weak-and StrongSegregation Block Copolymer Theories. Macromolecules 1996, 29, 1091-1098.

(30) Bates, C. M.; Maher, M. J.; Janes, D. W.; Ellison, C. J.; Willson, C. G. Block Copolymer Lithography. Macromolecules 2014, 47, 2-12.

(31) Miyake, Y.; Nagata, T.; Tanaka, H.; Yamazaki, M.; Ohta, M.; Kokawa, R.; Ogawa, T. Entropy-Controlled 2D Supramolecular Structures of N, N'-Bis (n-Alkyl) Naphthalenediimides on a HOPG Surface. ACS Nano 2012, 6, 3876-3887.

(32) Kleiner-Shuhler, L.; Brittain, R.; Johnston, M. R.; Hipps, K. W. Scanning Tunneling Microscopy and Orbital-Mediated Tunneling Spectroscopy of N, $\mathrm{N}^{\prime}$-Dioctyl-1,8:4,5-Naphthalenediimide Adsorbed on Highly Ordered Pyrolytic Graphite from Various Solvents and in Different Environments. J. Phys. Chem. C 2008, 112, 14907-14912.

(33) Owen, M. J. The Surface Activity of Silicones: a Short Review. Ind. Eng. Chem. Prod. Res. Dev. 1980, 19, 97-103.

(34) Seo, J.; Lee, L. P. Effects on Wettability by Surfactant Accumulation/Depletion in Bulk Polydimethylsiloxane (PDMS). Sens. Actuators, B 2006, 119, 192-198.

(35) Brandrup, J.; Immergut, E. H.; McDowell, W. Polymer Handbook; Wiley: 1975.

(36) Subhash Latthe, S.; Gurav, A. B.; Maruti, C. S.; Shrikant Vhatkar, R. Recent Progress in Preparation of Superhydrophobic Surfaces: a Review. J. Surf. Eng. Mater. Adv. Technol. 2012, 2, 76-94.

(37) Bhushan, B.; Jung, Y. C.; Koch, K. Micro-, Nano-and Hierarchical Structures for Superhydrophobicity, Self-Cleaning and Low Adhesion. Philos. Trans. R. Soc., A 2009, 367, 1631-1672.

(38) Gezer, P. G.; Brodsky, S.; Hsiao, A.; Liu, G. L.; Kokini, J. L. Modification of the Hydrophilic/Hydrophobic Characteristic of Zein Film Surfaces by Contact with Oxygen Plasma Treated PDMS and Oleic Acid Content. Colloids Surf., B 2015, 135, 433-440.

(39) Bharathidasan, T.; Narayanan, T. N.; Sathyanaryanan, S.; Sreejakumari, S. S. Above 170 Water Contact Angle and Oleophobicity of Fluorinated Graphene Oxide Based Transparent Polymeric Films. Carbon 2015, 84, 207-213.

(40) Plass, K. E.; Grzesiak, A. L.; Matzger, A. J. Molecular Packing and Symmetry of Two-Dimensional Crystals. Acc. Chem. Res. 2007, 40, 287-293.

(41) Slater, A. G.; Beton, P. H.; Champness, N. R. Two-Dimensional Supramolecular Chemistry on Surfaces. Chem. Sci. 2011, 2, 14401448.

(42) Slater, A. G.; Perdigão, L. M. A.; Beton, P. H.; Champness, N. R. Surface-Based Supramolecular Chemistry Using Hydrogen Bonds. Acc. Chem. Res. 2014, 47, 3417-3427.

(43) Horcas, I.; Fernández, R.; Gómez-Rodríguez, J. M.; Colchero, J.; Gómez-Herrero, J.; Baro, A. M. WSXM: A software for scanning probe microscopy and a tool for nanotechnology. Rev. Sci. Instrum. 2007, 78, 013705 